\title{
Obstetric Hemorrhage Risk Associated with Novel COVID-19 Diagnosis from a Single-Institution Cohort in the United States
}

\author{
Michelle J. Wang, MD ${ }^{1}$ Melissa Schapero, MS, MRes, MPhil ${ }^{1}$ Ronald Iverson, MD ${ }^{1}$ \\ Christina D. Yarrington, MD ${ }^{1}$
}

${ }^{1}$ Department of Obstetrics and Gynecology, Boston Medical Center,
Boston, Massachusetts Am J Perinatol 2020;37:1411-1416.
Address for correspondence Michelle J. Wang, MD, Department of Obstetrics and Gynecology, Boston Medical Center, 85 East Concord Street Boston, MA 02118 (e-mail: michelle.wang2@bmc.org).

\begin{abstract}
Keywords

- quantitative blood loss

- obstetric hemorrhage

- SARS-CoV-2

- Pregnancy

Objective The study aimed to compare the quantitative blood loss (QBL) and hemorrhage-related outcomes of pregnant women with and without a coronavirus disease 2019 (COVID-19) diagnosis.

Study Design This retrospective cohort study of all live deliveries at Boston Medical Center between April 1, 2020 and July 22, 2020 compares the outcomes of pregnant women with a laboratory-confirmed COVID-19 positive diagnosis and pregnant women without COVID-19. The primary outcomes are QBL and obstetric hemorrhage. The secondary outcomes analyzed were a maternal composite outcome that consisted of obstetric hemorrhage, telemetry-level (intermediate care unit) or intensive care unit, transfusion, length of stay greater than 5 days, or intraamniotic infection, and individual components of the maternal composite outcome. Groups were compared using Student's t-test, Chi-squared tests, or Fisher's exact. Logistic regression was used to adjust for confounding variables.

Results Of 813 women who delivered a live infant between April 1 and July 22, 2020, 53 women were diagnosed with COVID-19 on admission to the hospital. Women with a COVID-19 diagnosis at their time of delivery were significantly more likely to identify as a race other than white $(p=0.01)$, to deliver preterm $(p=0.05)$, to be diagnosed with preeclampsia with severe features $(p<0.01)$, and to require general anesthesia $(p<0.01)$. Women diagnosed with COVID-19 did not have a significantly higher QBL $(p=0.64)$. COVID-19 positive pregnant patients had no increased adjusted odds of obstetric hemorrhage (adjusted odds ratio [aOR]: $0.41,95 \%$ confidence interval [Cl]: $0.17-1.04$ ) and no increased adjusted odds of the maternal morbidity composite (aOR: $0.98,95 \% \mathrm{Cl}: 0.50-1.93)$ when compared with those without a diagnosis of COVID-19. Conclusion Pregnant women with COVID-19 diagnosis do not have increased risk for obstetric hemorrhage, increased QBL or risk of maternal morbidity compared with pregnant women without a COVID-19 diagnosis. Further research is needed to describe the impact of a COVID-19 diagnosis on maternal hematologic physiology and pregnancy outcomes.
\end{abstract}

received

August 12, 2020

accepted after revision

September 7, 2020

published online

September 29, 2020
Copyright $\odot 2020$ by Thieme Medical Publishers, Inc., 333 Seventh Avenue, New York, NY 10001, USA. Tel: +1(212) 760-0888.
DOI https://doi.org/

10.1055/s-0040-1718403. ISSN $0735-1631$. 


\section{Key Points}

- Information about blood loss associated with peripartum COVID-19 is limited.

- COVID-19 diagnosis is not associated with increase in obstetric hemorrhage.

- COVID-19 diagnosis is not associated with increase in blood loss.

Early case reports in the general population have demonstrated severe morbidities from coagulopathies and bleeding disorders associated with novel coronavirus disease 2019 (COVID-19) infection. ${ }^{1-3}$ Based on cases in pregnancy, it has been suggested that exacerbation of the immunologic shift of pregnancy combined with the cytokine storm provoked by COVID-19 infection could hasten consumptive coagulopathy. ${ }^{4}$ However, with very few studies reporting the blood loss associated with COVID-19 deliveries, there is a void of robust data to direct clinical practice. ${ }^{5,6}$

Our institution has well-established practice utilizing quantitative blood loss (QBL) in delivery and operating rooms. We sought to assess whether QBL and rate of obstetric hemorrhage were different among pregnant women with and without COVID-19 infection.

\section{Materials and Methods}

This was a retrospective cohort study of all live deliveries at Boston Medical Center, an urban academic medical center and the largest safety-net hospital in New England, between April 1, 2020 and July 22, 2020. Demographic, obstetric, delivery, and COVID-19 status information were abstracted. Institutional review board approval was obtained from Boston University School of Medicine.

At our institution, QBL measurement is collected for all deliveries. Blood loss is quantified in cesarean with calibrated suction canisters and in vaginal deliveries with calibrated under-buttocks drapes along with weighing blood-soaked materials. ${ }^{7}$ Information was gathered from department birth logs that are abstracted daily from the electronic medical record for all deliveries. This includes information about maternal demographics, maternal medical and obstetric history, antenatal course, intrapartum course, anesthesia type if any, and QBL. Additional information was abstracted by a trained chart abstractor (M.J.W.) into a standardized chart abstraction form using a secure database. Chart review was limited to the presenting symptoms and clinical course of COVID-19 infection among parturients.

Women were classified by COVID-19 status: positive or negative. Positive COVID-19 diagnosis was based on the result of a nasopharyngeal swab collected during that hospital admission. Our institution implemented universal testing on May 5, 2020. Prior to the implementation of universal testing, all patients were screened with questions recommended by the state department of public health and a temperature check but were only swabbed based on clinical suspicion.

Patients were also classified by presenting COVID-19 symptoms per national institutes of health guidelines as follows $^{8,9}$ :
- Asymptomatic or presymptomatic: Individuals who test positive for severe acute respiratory syndrome coronavirus 2 (SARS-CoV-2) by virologic testing using a molecular diagnostic (e.g., polymerase chain reaction) or antigen test, but have no symptoms.

- Mild illness: Individuals who have any of the various signs and symptoms of COVID-19 (e.g., fever, cough, sore throat, malaise, headache, and muscle pain) without shortness of breath, dyspnea, or abnormal chest imaging.

- Moderate Illness: Individuals who have evidence of lower respiratory disease by clinical assessment or imaging and a saturation of oxygen $(\mathrm{SpO} 2) \geq 94 \%$ on room air at sea level.

- Severe illness: Individuals who have respiratory frequency $>30$ breaths per minute, $\mathrm{SpO} 2<94 \%$ on room air at sea level, ratio of arterial partial pressure of oxygen to fraction of inspired oxygen $(\mathrm{PaO} 2 / \mathrm{FiO} 2)<300 \mathrm{~mm} \mathrm{Hg}$, or lung infiltrates $>50 \%$.

- Critical illness: Individuals who have respiratory failure, septic shock, and/or multiple organ dysfunction.

We chose to also include an additional stratification limited to two categories (asymptomatic or mild compared with moderate, severe, or critical) to stratify morbidity risks within the COVID-19 positive group.

The primary outcomes were QBL and obstetric hemorrhage. Obstetric hemorrhage was defined as any QBL greater than $1,000 \mathrm{~mL}$. The secondary outcome was a maternal morbidity composite based on existing maternal composites in the literature. ${ }^{10,11}$ This maternal composite consists of physiologic antecedents and consequences of obstetric hemorrhage and includes obstetric hemorrhage, telemetry-level (intermediate care unit) or intensive care unit stay, transfusion, length of stay greater than 5 days, or intraamniotic infection.

Exposure groups were compared with descriptive and bivariate statistics using Student's t-test for continuous variables, Chi-squared test, or Fisher's exact for categorical variables. Multiple logistic regression models for the primary and secondary outcomes were developed to estimate the effect of COVID-19 status on blood loss, hemorrhage, and other obstetric outcomes. Clinically relevant covariates for initial inclusion in multivariable statistical models were selected using results of the stratified analyses, and factors were removed in a backward stepwise fashion, based on significant changes in the exposure adjusted odds ratio. All analyses were completed using STATA MP, version 16 (College Station, TX).

\section{Results}

Of 813 women who delivered a live infant between April 1 and July 22, 2020, 53 women were diagnosed with COVID-19 
with a nasopharyngeal swab on admission to the hospital. Maternal and obstetric characteristics according to COVID19 status are shown in -Table 1. Exposure groups were not significantly different with regards to age, insurance type (private vs. public), body mass index (BMI), parity, delivery mode, or percentage of inductions. Women with a COVID-19 diagnosis were, however, more likely to be delivered preterm ( $<37$ weeks' gestation) ( $p=0.05)$, more likely to identify as
Hispanic race $(p<0.01)$, less likely to identify as White race ( $p=0.01$ ), and more likely to have a diagnosis of preeclampsia with severe features $(p<0.01)$ compared with women without a diagnosis of COVID-19. Despite no differences in delivery mode, women with COVID-19 were also more likely to receive general anesthesia $(p<0.01)$ and less likely to receive epidural anesthesia $(p=0.02)$ compared with women without a COVID-19 diagnosis.

\begin{tabular}{|c|c|c|c|}
\hline & $\begin{array}{l}\text { COVID positive } \\
\text { deliveries }(n=53)\end{array}$ & $\begin{array}{l}\text { COVID negative } \\
\text { deliveries }(n=760)\end{array}$ & $p$-Value \\
\hline Age & $29.8 \pm 5.9$ & $30.2 \pm 6.0$ & 0.63 \\
\hline \multicolumn{4}{|l|}{ Race } \\
\hline Hispanic & $-19(73.1)$ & $-119(39.8)$ & $<0.01$ \\
\hline Black & $-8(26.9)$ & $-111(37.1)$ & 0.61 \\
\hline White & $-0(0)$ & $-56(18.7)$ & 0.01 \\
\hline Asian & $-0(0)$ & $-4(1.3)$ & 0.29 \\
\hline Middle Eastern & $-0(0)$ & $-9(3.0)$ & 0.36 \\
\hline Government insurance & 45 (84.9) & $555(73.0)$ & 0.06 \\
\hline BMI $(n=812)$ & $33.1 \pm 7.2(n=53)$ & $32.4 \pm 6.3(n=758)$ & 0.42 \\
\hline $\mathrm{BMI}>30$ & $-36(67.9)$ & $-479(63.0)$ & -0.47 \\
\hline $\mathrm{BMI}>40$ & $-8(15.1)$ & $-86(11.3)$ & -0.41 \\
\hline \multicolumn{4}{|l|}{ COVID severity } \\
\hline Asymptomatic or mild & $-45(84.9)$ & & \\
\hline Moderate or severe or critical & $-8(15.1)$ & & \\
\hline \multicolumn{4}{|l|}{ COVID severity $(\mathrm{NIH})^{\mathrm{a}}$} \\
\hline Asymptomatic/presymptomatic & $-38(71.7)$ & & \\
\hline Mild illness & $-7(13.2)$ & & \\
\hline Moderate illness & $-2(3.8)$ & & \\
\hline Severe illness & $-4(7.6)$ & & \\
\hline Critical illness & $-2(3.8)$ & & \\
\hline Gestational age & $38.1 \pm 2.9$ & $38.9 \pm 2.1$ & $<0.01$ \\
\hline Preterm & $9(17.0)$ & $66(8.7)$ & 0.05 \\
\hline Parity & $2.9 \pm 1.6$ & $2.8 \pm 1.8$ & 0.53 \\
\hline Induction & $17(32.1)$ & $255(33.6)$ & 0.83 \\
\hline \multicolumn{4}{|l|}{ Delivery mode } \\
\hline Vaginal & $-30(56.6)$ & $-503(66.2)$ & 0.16 \\
\hline Cesarean & $-23(43.4)$ & $-257(33.8)$ & \\
\hline Preeclampsia with severe features & $10(18.9)$ & $59(7.8)$ & $<0.01$ \\
\hline \multicolumn{4}{|l|}{ Anesthesia type } \\
\hline None & $-14(26.4)$ & $-189(24.9)$ & 0.80 \\
\hline Epidural & $-19(35.9)$ & $-400(52.6)$ & 0.02 \\
\hline Spinal & $-10(18.9)$ & $-85(11.2)$ & 0.09 \\
\hline Combined & $-8(15.1)$ & $-82(10.8)$ & 0.33 \\
\hline General & $-2(3.8)$ & $-4(0.5)$ & $<0.01$ \\
\hline
\end{tabular}

Abbreviations: BMI, body mass ; COVID, coronavirus disease; NIH, national institutes of health.

Note: Percent of patients in that delivery mode category with the associated outcome or as mean \pm standard deviation.

${ }^{a}$ COVID severity defined per National Institutes of Health guidelines: https://www.covid19treatmentguidelines.nih.gov/overview/management-ofcovid-19/. 
Table 2 Maternal outcomes by COVID-19 status

\begin{tabular}{|c|c|c|c|c|}
\hline & $\begin{array}{l}\text { COVID positive } \\
\text { deliveries }(n=53)\end{array}$ & $\begin{array}{l}\text { COVID negative } \\
\text { deliveries }(n=760)\end{array}$ & aOR $(95 \% \mathrm{Cl})$ & $p$-Value \\
\hline Quantitative blood loss & $551 \pm 332.2$ & $584.7 \pm 509.3$ & & 0.64 \\
\hline Obstetric hemorrhage & $6(11.3)$ & $140(18.4)$ & $0.41(0.17-1.04)^{c}$ & \\
\hline Maternal composite ${ }^{a}$ & $17(32.1)$ & $205(27.0)$ & $0.98(0.50-1.93)^{b}$ & \\
\hline Intraamniotic infection & $2(3.8)$ & $46(6.1)$ & $0.61(0.14-2.57)$ & \\
\hline Any transfusion & $0(0)$ & $14(1.8)$ & & 0.32 \\
\hline Maternal LOS & $4.4 \pm 4.0$ & $3.4 \pm 1.8$ & & $<0.01$ \\
\hline $\operatorname{LOS}>5 d$ & $12(22.6)$ & $74(9.7)$ & $1.8(0.73-4.61)^{\mathrm{d}}$ & \\
\hline ICU or IMCU & $6(11.3)$ & $5(0.7)$ & $28.5(6.73-120.19)^{\mathrm{e}}$ & \\
\hline
\end{tabular}

Abbreviations: aOR, adjusted odds ratio; BMI, body mass values; COVID, coronavirus disease; $\mathrm{Cl}$, confidence interval; ICU, intensive care unit; IMCU, intermediate care unit; LOS, length of stay.

${ }^{a}$ Maternal composite outcome is considered categorically positive for maternal morbidity if the mother experienced any of the following factors: (i) $\mathrm{OBH}$, (ii) ICU or IMCU stay, (iii) transfusion, (iv) length of stay greater than 5 days, or (v) intraamniotic infection.

${ }^{\mathrm{b}}$ Adjusted for delivery mode, BMI, and gestational age.

${ }^{\mathrm{C}}$ Adjusted for delivery mode, BMI, and intraamniotic infection.

${ }^{\mathrm{d} A d j u s t e d ~ f o r ~ d e l i v e r y ~ m o d e, ~ I C U ~ o r ~ I M C U ~ a d m i s s i o n, ~ i n t r a a m n i o t i c ~ i n f e c t i o n, ~ a n d ~ g e s t a t i o n a l ~ a g e . ~}$

eAdjusted for delivery mode and obstetric hemorrhage.

Maternal outcomes according to COVID-19 status are shown in - Table 2. Women diagnosed with COVID-19 did not have a significantly higher $\mathrm{QBL}(p=0.64)$. After adjusting for delivery mode, BMI and intraamniotic infection, women with COVID-19 had no increased odds of obstetric hemorrhage compared with those without COVID-19 (adjusted odds ratio [aOR]: $0.41,95 \%$ confidence interval [CI]: 0.17-1.04). Furthermore, we observed no difference in the maternal composite outcome in COVID-19 positive patients compared with the women without a COVID-19 diagnosis after adjusting for delivery mode, BMI, and gestational age (aOR: 0.98, 95\% CI: 0.50-1.93).

Thirty-eight women (71.7\%) with a COVID-19 diagnosis admitted to the hospital during this time were asymptomatic or presymptomatic, seven $(13.2 \%)$ had mild illness, two (3.8\%) had moderate illness, four (7.6\%) had severe illness, and two (3.8) had critical illness. Eight (35\%) of COVID-19 cesarean deliveries were indicated for worsening COVID-19 related symptoms, six (26\%) for nonreassuring fetal heart tracing, five (22\%) were scheduled Cesareans, two (9\%) for preeclampsia with severe features, and two (9\%) for failure to progress. Presentation with moderate or severe COVID-19 symptoms was not associated with increased odds of obstetric hemorrhage (odds ratio [OR]: 2.2, 95\% confidence interval [CI]: 0.54-9.01), but was associated with increased odds of delivery by cesarean (OR: $12.3,95 \% \mathrm{CI}: 1.38-108.74$ ).

\section{Discussion}

Deliveries associated with a COVID-19 diagnosis were not found to have an increase in average QBL, risk of obstetric hemorrhage, or risk of maternal morbidity compared with non-COVID deliveries. While not a pre hoc outcome, our cohort did, however, demonstrate an increased proportion of non-White women and a rate of severe preeclampsia twofold higher in the COVID positive cohort compared with the COVID negative cohort.

Few studies have examined hemorrhage risk associated with COVID-19 in pregnancy, and no studies have published any data regarding the average blood loss associated with COVID-19 deliveries. Similar to the findings from our study, one early study from China found no difference in the incidence of postpartum hemorrhage in vaginal deliveries associated with COVID-19 positive compared with COVID-19 negative patients. ${ }^{5}$ Another study from China examining a COVID-19 positive cohort delivered via cesarean with no COVID-19 negative comparison group found no difference in risk of postpartum hemorrhage when comparing severity of COVID-19 disease, comparable to the findings from the secondary analysis in our present study. ${ }^{6}$

While some larger studies have examined in detail the medical course of hospitalized COVID-19 positive pregnant patients, few studies have focused on blood-loss and hemorrhage-related outcomes of COVID-19 in pregnancy. ${ }^{12-15}$ One case series found coagulopathy-indicating laboratory markers in two pregnant patients; only one of the patients had a delivery associated with postpartum hemorrhage. ${ }^{4}$ Furthermore, while reviews and case reports in nonpregnant populations have reported some severe morbidity and even mortality in COVID-19 associated with bleeding or hemorrhage, ${ }^{1-4}$ there have been very limited data regarding specific laboratory derangements that may be predictive for or associated with bleeding risk related to COVID-19. ${ }^{16-18}$ Further research should continue to investigate the impact of a COVID-19 diagnosis on hematologic physiology, and also its impact specifically in the setting of pregnancy.

The present data can help to guide obstetricians and maternal fetal medicine specialists in assessing hemorrhage risk of patients upon admission to labor and delivery. Early identification and frequent reassessment of risk factors can 
help with preparing interventions (e.g., type and cross, blood products, and uterotonics) and prepare the clinical team for increased surveillance following birth that all may help prevent adverse outcomes. ${ }^{19}$ As COVID-19 diagnosis is not presently associated with either a statistically increased QBL or an increased obstetric hemorrhage risk, we would not recommend adjusting hemorrhage risk assessment calculators based on COVID-19 status.

While demographic and comorbidity incidence were not our primary outcomes, we did have some compelling and concerning findings that are similar to findings in existing COVID-19 literature. In our cohort, there was a significantly higher proportion of pregnant women who identified as nonWhite with a COVID-19 diagnosis when compared with the COVID-19 negative cohort. Specifically, there was a significantly higher proportion of Hispanic women in our COVID-19 positive cohort compared with the COVID-19 negative cohort. This is aligned with many studies both in pregnant and nonpregnant populations that have demonstrated significant racial disparities in incidence and outcomes. ${ }^{13,20,21}$ A multitude of factors, including health care access, social determinants of health, and underlying comorbidities may underlie these disparities in COVID-19 spread, incidence, and outcomes in non-White populations. The etiology of these demographic findings should be interrogated seriously with both qualitative and quantitative approaches. ${ }^{22}$

Lastly, we also found that women with a COVID-19 diagnosis were also more likely to be diagnosed with preeclampsia with severe features. The relationship between COVID-19 diagnosis and hypertensive diseases of pregnancy such as preeclampsia has been an area of intense scrutiny as there are many confounding symptoms (e.g., shortness of breath or neurological manifestations such as headache or seizure) and laboratory abnormalities (e.g., thrombocytopenia, anemia, transaminitis) that these two diseases share. ${ }^{23,24}$ At institutions where there is not yet universal testing, for women who present with severe features of preeclampsia, COVID-19 must necessarily be high on the differential and women should be tested and appropriately treated for either disease or possibly both. Further research, both clinical and pathology based, is needed to further explore the relationship between these two diseases.

Our study has several strengths. To date, ours is the first study to specifically address blood loss and hemorrhage in an American population of COVID-19 positive and negative parturients. Furthermore, we were able to present QBL, a more precise measure of hemorrhage than traditional estimated blood loss. ${ }^{25,26}$ Most of the limited literature in this field have relied large national cohorts to describe obstetric and surgical morbidity in the setting of COVID-19 infection. ${ }^{12-15}$ These databases necessarily combine results from institutions with varying protocols for hemorrhage risk screening and management. Additionally, multicohort studies are limited by misclassification biases secondary to significant variance in study setting. As a single institution cohort study, the patients described here were treated with one set of protocols from our institution; thus, our data are subject to less intersubject variance.
We acknowledge our study limitations as a nonrandomized cohort with unequal sample sizes of our comparison groups. While we attempted to control for known differences between the groups, residual confounding may persist. Furthermore, the utilization of a categorical variable of obstetric hemorrhage, defined by our U.S. national guidelines as a relatively arbitrary threshold of $1,000 \mathrm{~mL}$ for poor bloodloss outcomes may not be as predictive or clinically significant as other factors, such as laboratory derangements or hemorrhage treatments utilized. ${ }^{27}$ National guidelines on obstetric hemorrhage vary significantly and thus influence treatment specific protocols, which may make international multicohort studies and large-scale meta-analyses difficult to interpret. ${ }^{28}$ We are hopeful, however, that our data may contribute to the larger understanding of the interaction of COVID-19 infection and obstetric hematologic physiology.

\section{Conclusion}

Mothers with COVID-19 diagnosis were not found to have an increased risk in hemorrhagic morbidity or increased risk of overall maternal morbidity. However, as hemorrhage persists as the leading etiology of severe maternal morbidity and mortality in the United States, obstetricians should continue to practice with clinical vigilance in providing care for the COVID-19 infected parturient as we continue to learn more about the perinatal sequelae of this disease.

Note

This study is approved by Boston University Institutional Reference Board (reference number: 1311956).

Conflict of Interest

None declared.

\section{References}

1 Tang N, Li D, Wang X, Sun Z. Abnormal coagulation parameters are associated with poor prognosis in patients with novel coronavirus pneumonia. J Thromb Haemost 2020;18(04):844-847

2 Dorgalaleh A. Bleeding and Bleeding Risk in COVID-19. Semin Thromb Hemost 2020

3 Al-Samkari H, Karp Leaf RS, Dzik WH, et al. COVID-19 and coagulation: bleeding and thrombotic manifestations of SARSCoV-2 infection. Blood 2020;136(04):489-500

4 Vlachodimitropoulou Koumoutsea E, Vivanti AJ, Shehata N, et al. COVID-19 and acute coagulopathy in pregnancy. J Thromb Haemost 2020;18(07):1648-1652

5 Liao J, He X, Gong Q, Yang L, Zhou C, Li J. Analysis of vaginal delivery outcomes among pregnant women in Wuhan, China during the COVID-19 pandemic. Int J Gynaecol Obstet 2020;150 (01):53-57

6 Zhang L, Jiang Y, Wei M, et al. [Analysis of the pregnancy outcomes in pregnant women with COVID-19 in Hubei Province]. Zhonghua Fu Chan Ke Za Zhi 2020;55(03):166-171

7 Lyndon A, McNulty J, Vanderwal B, et al. Cumulative quantitative assessment of blood loss. California Maternal Quality Care Collaborative Obstetric Hemorrhage Toolkit Version 2.0 Available at: https://www.cmqcc.org/resource/ob-hem-cumulative-quantitative-assessment-blood-loss Accessed June 1, 2020

8 “Management of COVID-19." National Institutes of Health, U.S. Department of Health and Human Services, Updated 11 
June 2020. Available at: www.covid19treatmentguidelines.nih. gov/overview/management-of-covid-19/. Accessed August 22, 2020

9 Wu Z, McGoogan JM. Characteristics of and important lessons from the coronavirus disease 2019 (COVID-19) outbreak in China: summary of a report of 72314 cases from the chinese center for disease control and prevention. JAMA 2020;323(13):1239-1242

10 Rossi RM, DeFranco EA. Maternal complications associated with periviable birth. Obstet Gynecol 2018;132(01):107-114

11 Kuper SG, Sievert RA, Steele R, Biggio JR, Tita AT, Harper LM. Maternal and neonatal outcomes in indicated preterm births based on the intended mode of delivery. Obstet Gynecol 2017; 130(05):1143-1151

12 Pierce-Williams RAM, Burd J, Felder L, et al. Clinical course of severe and critical COVID-19 in hospitalized pregnancies: a US cohort study. Am J Obstet Gynecol MFM. 2020;2(03):100134

13 Knight M, Bunch K, Vousden N, et al; UK Obstetric Surveillance System SARS-CoV-2 Infection in Pregnancy Collaborative Group. Characteristics and outcomes of pregnant women admitted to hospital with confirmed SARS-CoV-2 infection in UK: national population based cohort study. BMJ 2020;369:m2107

14 Kayem G, Lecarpentier E, Deruelle P, et al. A snapshot of the COVID-19 pandemic among pregnant women in France. J Gynecol Obstet Hum Reprod 2020;49(07):101826

15 Martínez-Perez O, Vouga M, Cruz Melguizo S, et al. Association between mode of delivery among pregnant women with COVID19 and maternal and neonatal outcomes in Spain. JAMA 2020;324 (03):296-299

16 Levi M, Thachil J, Iba T, Levy JH. Coagulation abnormalities and thrombosis in patients with COVID-19. Lancet Haematol 2020;7 (06):e438-e440

17 Connors JM, Levy JH. COVID-19 and its implications for thrombosis and anticoagulation. Blood 2020;135(23):2033-2040
18 Benhamou D, Keita H, Ducloy-Bouthors ASCARO working group. Coagulation changes and thromboembolic risk in COVID-19 obstetric patients. Anaesth Crit Care Pain Med 2020;39(03): 351-353

19 Gabel K, Lyndon A, Main E. OB hemorrhage risk factor assessment. California maternal quality care collaborative obstetric hemorrhage toolkit version 2.0 Available at: https://www.cmqcc.org/resource/ob-hem-risk-factor-assessment. Accessed June 01, 2020

20 Khunti K, Singh AK, Pareek M, Hanif W. Is ethnicity linked to incidence or outcomes of covid-19? BMJ 2020;369:m1548

21 Price-Haywood EG, Burton J, Fort D, Seoane L. Hospitalization and mortality among black patients and white patients with COVID19. N Engl J Med 2020;382(26):2534-2543

22 Bryant AS, Worjoloh A, Caughey AB, Washington AE. Racial/ethnic disparities in obstetric outcomes and care: prevalence and determinants. Am J Obstet Gynecol 2010;202(04):335-343

23 Abbas AM, Ahmed OA, Shaltout AS. COVID-19 and maternal preeclampsia: a synopsis. Scand J Immunol 2020;92(03):e12918

24 Gidlöf S, Savchenko J, Brune T, Josefsson H. COVID-19 in pregnancy with comorbidities: more liberal testing strategy is needed. Acta Obstet Gynecol Scand 2020;99(07):948-949

25 Bose P, Regan F, Paterson-Brown S. Improving the accuracy of estimated blood loss at obstetric haemorrhage using clinical reconstructions. BJOG 2006;113(08):919-924

26 Della Torre M, Kilpatrick SJ, Hibbard JU, et al. Assessing preventability for obstetric hemorrhage. Am J Perinatol 2011;28(10): 753-760

27 Kerr RS, Weeks AD. Postpartum haemorrhage: a single definition is no longer enough. BJOG 2017;124(05):723-726

28 Dahlke JD, Mendez-Figueroa H, Maggio L, et al. Prevention and management of postpartum hemorrhage: a comparison of 4 national guidelines. Am J Obstet Gynecol 2015;213(01):76.e1-76.e10 